\title{
Sensory hybrid host materials for the selective chromo-fluorogenic detection of biogenic amines
}

\author{
Beatriz García-Acosta, ${ }^{a}$ María Comes, ${ }^{a}$ Julia L. Bricks, ${ }^{* b}$ Margarita A. Kudinova, ${ }^{b}$ Vladimir V. Kurdyukov, ${ }^{b}$ \\ Alexei I. Tolmachev, ${ }^{b}$ Ana B. Descalzo,${ }^{a c}$ M. Dolores Marcos, ${ }^{a}$ Ramón Martínez-Máñez, ${ }^{* a}$ Ana Moreno, ${ }^{a}$ \\ Félix Sancenón, ${ }^{a}$ Juan Soto, ${ }^{a}$ Luis A. Villaescusa, ${ }^{a}$ Knut Rurack, ${ }^{* c}$ José M. Barat, ${ }^{d}$ Isabel Escriche $^{d}$ and \\ Pedro Amorós ${ }^{e}$
}

Received (in Cambridge, UK) 20th February 2006, Accepted 6th April 2006

First published as an Advance Article on the web 2nd May 2006

DOI: $10.1039 / \mathrm{b} 602497 \mathrm{a}$

Pyrylium-containing mesoporous materials have been used for the chromo-fluorogenic sensing of biogenic amines in an aqueous environment.

Biologically active amines are ubiquitous chemical compounds that play an important role in many different areas. Depending on their chemical composition and origin, basically two different groups can be found. A major group are the so-called biogenic amines that are formed in the normal metabolism of animals, plants and micro-organisms via decarboxylation of the respective amino acid through substrate-specific enzymatic reactions. The formation of these biogenic amines is undesirable in foods and beverages because they can induce headaches, respiratory distress, heart palpitations and several allergenic disorders. ${ }^{1}$ Histamine is the most toxic of the biogenic amines and the main cause of scombroid poisoning by acting synergistically with other amines. Thus, the level of biogenic amines in a food product is a quality index and the development of easy-to-use determination protocols is of interest. The second group are fatty amines such as sphingosine (a fatty amine that carries two additional hydroxyl groups in the polar head region) and its close metabolites that are important cellular messengers and have a profound impact on e.g. mitosis and apoptosis. ${ }^{2}$ Other fatty amines, that usually stem form anthropogenic sources, are simple amines with a chain length $>\mathrm{C}_{10}$ that find wide-spread application in corrosion inhibition, asphalt emulsions or as petroleum additives and are well known for their antifungal and disinfectant properties. ${ }^{3}$ Accordingly, the toxicological role of the latter group of compounds has the largest impact on aquatic organisms and, for instance in marine foodstuffs, a combination of all these amines can be encountered.

All these amines are usually determined by chromatographic methods. However, these techniques are costly and not suitable for in situ sensing or rapid screening applications. An appealing

${ }^{a}$ Instituto de Química Molecular Aplicada, Departamento de Química, Universidad Politécnica de Valencia, Camino de Vera s/n, E-46071 Valencia,Spain.E-mail: rmaez@qim.upv.es

${ }^{b}$ Institute of Organic Chemistry, National Academy of Sciences, 5 Murmanskaya St., Kiev-94, UKR-253660, Ukraine.

E-mail:timophei@bricks.kiev.ua

${ }^{c}$ Div. I.5, Bundesanstalt für Materialforschung und-prüfung (BAM), Richard-Willstätter-Strasse 11, D-12489 Berlin, Germany.

E-mail: knut.rurack@bam.de

${ }^{d}$ Departamento de Tecnología de Alimentos, Universidad Politécnica de Valencia, Camino de Vera s/n, E-46022 Valencia, Spain

${ }^{e}$ Institut de Ciència del Materials (ICMUV), Universitat de Valencia,

P.O. Box 2085, E-46071 Valencia, Spain alternative is the use of selective chromo- or fluorogenic chemosensors. ${ }^{4}$ In fact the development of highly selective probes or indicators for small bio-molecules is still a barely studied field.

One such advanced goal is the development of selective probes for real samples that would sense biogenic amines (first group, see above) via a simple chromo-fluorogenic test but would ideally remain silent in the presence of fatty amines (second group) and amino acids (another prominent source of amino moieties).

To achieve this goal we focused our attention on pyrylium compounds. As we and others have shown previously, such heterocyclic ring systems react with amines to give the corresponding pyridinium derivatives. ${ }^{5}$ Furthermore, pyrylium and the respective pyridinium salts are strong electron acceptors so that appropriate integration into a charge-transfer chromophore yields deeply coloured dyes that absorb and emit in the red spectral range. ${ }^{6}$

The compounds P1-P7 (pyrylium dyes) and M1-M7 (methylpyridinium derivatives) (see Scheme 1) were prepared ${ }^{7}$ and investigated to select the optimum combination of pyryliummethylpyridinium dye for the selective and sensitive detection of

A)
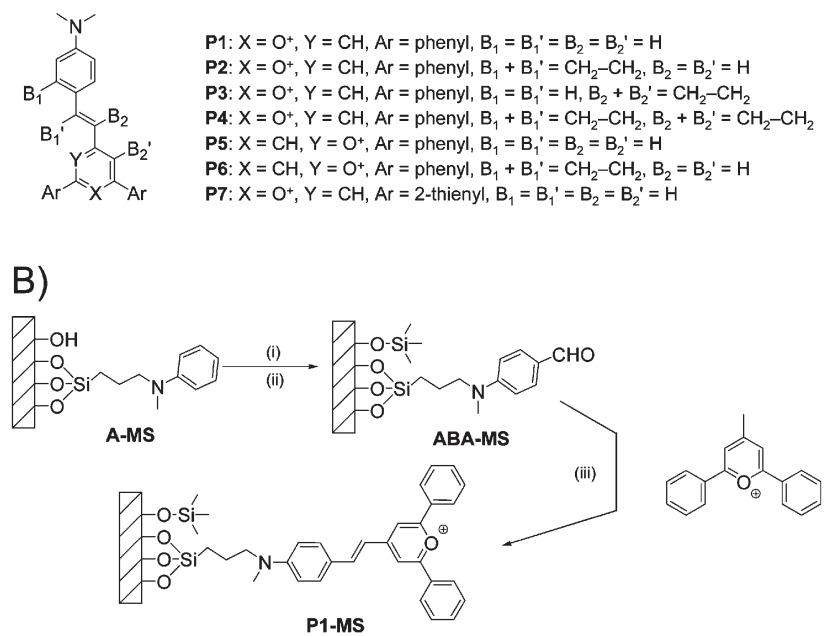

Scheme 1 (A) Compound library of pyrylium dyes spectroscopically studied and tested as sensor molecules for biologically active amines. The corresponding methylpyridinium derivatives $\mathbf{M 1 - M 7}$ with $\mathrm{N}^{+}-\mathrm{CH}_{3}$ instead of $\mathrm{O}^{+}$have been also studied spectroscopically. (B) Synthesis of solid P1-MS. (i) Hexamethyldisilizane, toluene, room temperature, $16 \mathrm{~h}$, (ii) $\mathrm{POCl}_{3}$ in $\mathrm{DMF} 1 \mathrm{~h}$ at $0{ }^{\circ} \mathrm{C}$ and then $3 \mathrm{~h}$ at $100{ }^{\circ} \mathrm{C}$, (iii) 4-methyl-2,6diphenylpyrylium, $\mathrm{MeCN}, 80{ }^{\circ} \mathrm{C}, 16 \mathrm{~h}$. 
the target analytes. While all the $\mathbf{P}$ dyes absorb at $c a .640 \mathrm{~nm}$ in highly polar solvents, their different bridging patterns and positions of the reactive oxygen atom were assumed to influence selectivity. The decisive criterion for the $\mathbf{M}$ dyes, that serve as models for the product of the sensing reaction between amine and $\mathbf{P}$ dye, lies with signal output. Whereas the emission of differently rigidized styryl dyes is commonly found at similar wavelengths, their fluorescence yields often depend dramatically on the flexibility of the $\pi$ system. ${ }^{8}$ Spectroscopic studies of the dyes revealed that three of the methylpyridinium dyes (M1, M2, M4) show a reasonable fluorescence of $0.05>\Phi_{\mathrm{f}}>5 \times 10^{-3}$ in $\mathrm{MeCN}$ in the red spectral range, at $\lambda_{\max } \sim 660 \mathrm{~nm}$.

However, concerning a unique preference for biologically active amines, all the $\mathbf{P}$ dyes are rather unselective and thus not suitable as molecular sensors. At room temperature, they react with biogenic amines such as histamine, putrescine and cadaverine as well as with decylamine (as a representative of large lipophilic fatty amines) in mixed aqueous organic solutions $\left(\mathrm{H}_{2} \mathrm{O}: \mathrm{MeCN}\right.$ $90: 10 \mathrm{v} / \mathrm{v}$ ) at basic $\mathrm{pH}$, but also with a number of natural amino acids, especially glycine, tyrosine, histidine, cysteine and lysine. As the best performance in this respect was found for P1 and since M1 is among the most fluorescent $\mathbf{M}$ derivatives, the pair P1-M1 was selected for further improvement of the system.

A way to overcome a lack of selectivity is to enhance host-guest interactions for the particular target analyte. Apart from the traditional design of complex hosts with multiple binding sites, enhanced selectivity can be obtained by coupling the probe to nanoscopic inorganic solids. Here, the use of mesoporous solids is highly suitable because, if properly functionalised, their rigid 3D nanoscopic scaffold can behave as a biomimetic prototype of "binding pockets" of bio-molecules. 9 Thus, to improve the analytical performance of the couple P1-M1 we anchored P1 covalently to the inner walls of the hydrophobically functionalised mesopores of a disordered MCM-41 type ${ }^{10}$ hybrid material (Scheme 1). ${ }^{11}$

For comparison and to demonstrate the effect of the functionalised "binding pockets" on the final selectivity observed, two more systems were prepared; (i) the P1-FS solid containing P1 probes anchored onto hydrophobized fumed silica (similar to P1-MS but without the presence of mesopores) and (ii) a sensory PVC-membrane containing the dye P1 and NPOE as plasticizer (P1-PVC). ${ }^{12}$

The performance of these materials was tested in water at $\mathrm{pH} 10.5$ in the presence of biogenic amines and natural amino acids as potential competitors. The reaction of the blue materials $\left(\lambda_{\max }=650 \mathrm{~nm}\right)$ with histamine, putrescine and cadaverine resulted in the evolution of a new band at $521 \mathrm{~nm}$ due to the formation of the corresponding pyridinium derivative (colour modulation from blue to red) within a few minutes for upper millimolar concentrations. Although lower concentrations require longer reaction times, the latter can be satisfactorily shortened by heating. As an example, Fig. 1 shows a photograph of solid P1-MS in the presence of amino acids and biogenic amines, whereas Fig. 2 shows the fluorometric sensing features that allow, using relatively simple devices, a sensitive detection even at low biogenic amine concentration when the chromo-fluorogenic reaction is only partially completed. The emission of the reaction product can be conveniently monitored in the red visible range, the relationship between analyte concentration and response is linear, and the

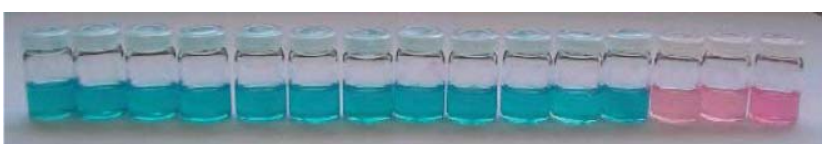

Fig. 1 Photograph showing the colour change in P1-MS in the presence of amino acids and biogenic amines. From left to right: no guest, glycine, valine, leucine, phenylalanine, proline, tyrosine, arginine, tryptophan, cysteine, lysine, histidine, histamine, putrescine and cadaverine. The final solids filtered and suspended in ethylene glycol are shown.

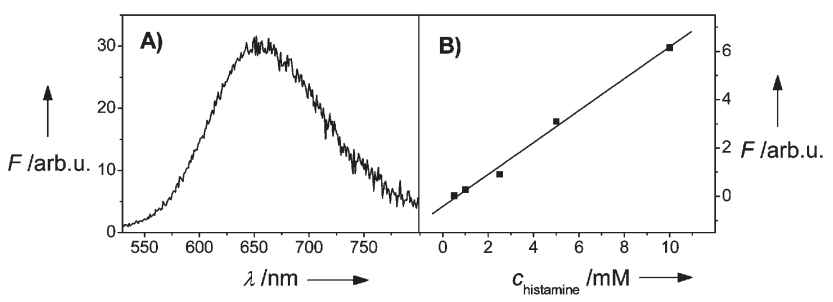

Fig. 2 (A) Emission spectrum of the P1-MS-histamine reaction product as measured for $0.01 \mathrm{mg} \mathrm{mL}^{-1}$ solid suspended in ethylene glycol; $\lambda_{\mathrm{exc}}=$ 490 nm. (B) Corresponding calibration curve.

combination of the sensory materials (here P1-MS) with fluorometric detection is sufficiently sensitive to yield a limit of detection of $5 \times 10^{-4} \mathrm{M}$ of histamine.

As it is shown exemplarily in Fig. 1, there is a remarkably selective response to biogenic amines, whereas P1-FS, P1-MS and P1-PVC remain completely silent even in the presence of a very large excess of amino acids. Especially the discrimination of histamine over its closely related parent amino acid histidine and other amino acids with nucleophilic residues (e.g. lysine and cysteine) is a striking feature and clearly shows the improved response of the prepared sensory materials when compared to that of P1 in solution under similar conditions (see above). Apparently, the confinement of the probe into hydrophobic systems protects the anchored P1 molecules from a nucleophilic attack of charged amino acids, i.e. hinders the amino acids to enter or approach the hydrophobic environments, while the neutral amines can still react.

This discrimination of biogenic amines vs. amino acids is very similar for the three sensory materials P1-FS, P1-MS and P1PVC. However, they behave differently if discrimination by size and lipophilicity is considered. For instance, this differentiation is evident from a comparison of the reactivity of the solids with putrescine and a fatty amine such as $n$-decylamine. Here, P1-PVC reacts faster with $n$-decylamine than with putrescine, whereas in remarkable contrast the solid P1-MS displays a much faster reactivity for putrescine than for $n$-decylamine (similar results were obtained with histamine). This control in the selectivity can be seen in Fig. 3 that shows the colour change (absorbance at $520 \mathrm{~nm}$ ) in the presence of $300 \mathrm{ppm}$ of putrescine or $n$-decylamine in water for the sensory materials P1-MS and P1-PVC. Finally, P1-FS displays a rather similar reactivity for both amines (not shown).

This behaviour is tentatively attributed to the individual features of the different solids. P1-PVC, a highly disordered material with a large distribution of pore sizes, is clearly preferred by the fatty amines due to the highest lipophilic character of the three hybrids. P1-FS, conglomerates of high-surface nanoparticles with a textural porosity, are largely indifferent toward the lipophilicity of the guest. On the other hand, the size discrimination observed 

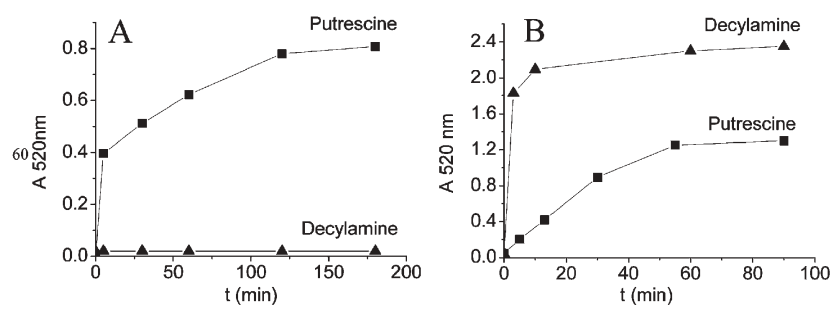

Fig. 3 Absorbance vs. time at $520 \mathrm{~nm}$ in the presence of $300 \mathrm{ppm}$ of putrescine or $n$-decylamine in water at $60{ }^{\circ} \mathrm{C}$ for the sensory materials. (A) P1-MS and (B) P1-PVC.

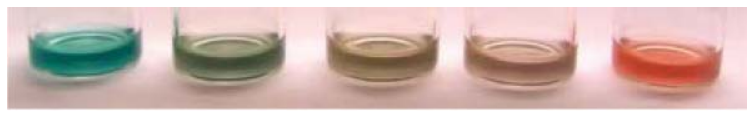

Fig. 4 Photograph of a suspension of solid P1-MS in contact with aqueous extracts of gilthead bream (S. aurata) at $\mathrm{pH} 10$ with increasing amounts of histamine after $30 \mathrm{~min}$ of reaction at $50^{\circ} \mathrm{C}$. From left to right: $c_{\text {histamine }}=0,3.75 \times 10^{-3}, 6.25 \times 10^{-3}, 1.25 \times 10^{-2}$ and $2.50 \times 10^{-2} \mathrm{M}$.

for the MCM41-type P1-MS solid is based on the interplay between the passivated surface that tends to prefer more hydrophobic guests and the size of the ordered and rather uniform pores that tend to discriminate by size. Furthermore, during the course of an analysis, increasing reaction events will block the entrances of the channels more effectively for long-chain amines. These trends lead to the fact that amines with a medium-sized alkylic or alkylarylic end group exhibit a much faster response than the fatty amines. The combination of molecular concepts and preorganized 3D solid state features thus display synergistic effects that allow one to modulate simple hydrophobic discrimination toward a more sophisticated differentiation by polarity and size. ${ }^{13}$

Motivated by the favourable response features of the sensory materials, we tested the potential of P1-MS for the determination of biogenic amines in realistic media, extracts of fish (Sparus aurata) spiked with increasing amounts of histamine. In a typical assay, $0.35 \mathrm{~mL}$ of an aqueous extract of $S$. aurata (obtained from $10 \mathrm{~g}$ of the fish ground up with $50 \mathrm{~mL}$ of water, heated and filtered or centrifuged) at $\mathrm{pH} 10$ were mixed with known amounts of histamine and $1 \mathrm{mg}$ of P1-MS. The gradual colorimetric modulation was observed as a function of histamine concentration (Fig. 4). This response, visible by the naked eye, from blue to red-orange in a complex realistic sample medium suggests that P1-MS, or similar systems, are promising optical sensors for the rapid screening of toxic biogenic amines in target foods.

In summary, we have shown the rational development of sensory materials for the chromo- and fluorogenic detection of biogenic amines in complex liquid samples. The probe molecule is a reactive pyrylium chromophore that is anchored into the inner hydrophobic pores of a mesoporous siliceous support. The solid P1-MS demonstrates that the combination of molecular concepts and 3D solid state preorganized features (for instance confinement in nanometric pores and hydrophobicity) might open new attractive and synergistic hetero-supramolecular routes to enhanced recognition/sensing protocols for species of interest.

We thank the Ministerio de Ciencia y Tecnología (MAT200308568-C03-02) for support. F.S. is thankful for a Ramón y Cajal contract. B.G.A. thanks the Generalitat Valenciana and M.C. thanks the Caja de Ahorros del Mediterraneo for a Doctoral Fellowship. A.B.D. thanks the Alexander-von-Humboldt Foundation for a Research Fellowship.

\section{Notes and references}

1 C. Ruiz-Capillas and F. Jiménez-Colmenero, Crit. Rev. Food Sci., 2004, 44, 489.

2 M. Maceyka, S. G. Payne, S. Milstien and S. Spiegel, Biochim. Biophys. Acta, 2002, 1585, 193.

3 J. A. Finlay and M. E. Callow, Biofouling, 1996, 9, 257.

4 R. Martínez-Máñez and F. Sancenón, Chem. Rev., 2003, 104, 4419.

5 A. I. Tolmachev, N. A. Derevyanko, E. F. Karaban and M. A. Kudinova, Khim. Geterotsikl. Soedin., 1975, 612; A. R. Katritzky, Tetrahedron, 1980, 36, 679; A. T. Balaban, A. Dinculescu, G. N. Dorofeenko, G. W. Fischer, A. V. Koblik, V. V. Mezheritskii and W. Schroth, Adv. Heterocycl. Chem, 1982, Suppl. 2, pp. 1-404.

6 J. L. Bricks, J. L. Slominskii, M. A. Kudinova, A. I. Tolmachev, K. Rurack, U. Resch-Genger and W. Rettig, J. Photochem. Photobiol., A, 2000, 132, 193.

7 For general synthesis details see: ref 6; M. Sczepan, W. Rettig, A. I. Tolmachev and V. V. Kurdyukov, Phys. Chem. Chem. Phys., 2001, 3, 3555; R. Wizinger and P. Ulrich, Helv. Chim. Acta, 1956, 39, 207; M. A. Kudinova, V. V. Kurdyukov and A. D. Kachkovskii, Khim. Geterotsikl. Soedin., 1998, 494. Additionally compound P6 was prepared by reaction of 2-methyl-4,6-diphenylpyrylium tetrafluoroborate and 5-dimethylamino-1-indanone (cf. A. T. Brown, G. Hallas and R. Lawson, Chem. Ind., 1981, 7, 248) in acetic anhydride at $125-130{ }^{\circ} \mathrm{C}$ for $1 \mathrm{~h}$.

8 M. Sczepan, W. Rettig and A. I. Tolmachev, Photochem. Photobiol. Sci., 2003, 2, 1264

9 A. B. Descalzo, K. Rurack, H. Weisshoff, R. Martínez-Máñez, M. D. Marcos, P. Amorós, K. Hoffman and J. Soto, J. Am. Chem. Soc., 2005, 127, 184; M. Comes, G. Rodríguez-López, M. D. Marcos, R. Martínez-Máñez, F. Sancenón, J. Soto, L. A. Villaescusa, P. Amorós and D. Beltrán, Angew. Chem., Int. Ed., 2005, 44, 2918; D. R. Radu, C.-Y. Lai, J. W. Wiench, M. Pruski and V. S.-Y. Lin, J. Am. Chem. Soc., 2004, 126, 1640; V. S.-Y. Lin, C.-Y. Lai, J. Huang, S.-A. Song and S. Xu, J. Am. Chem. Soc., 2001, 123, 11510; M. Comes, M. D. Marcos, R. Martínez-Máñez, F. Sancenón, J. Soto, L. A. Villaescusa, P. Amorós and D. Beltrán, Adv. Mater., 2004, 16, 1783.

10 J. El Haskouri, D. Ortíz de Zárate, C. Guillém, J. Latorre, M. Caldés, A. Beltrán, D. Beltrán, A. B. Descalzo, G. Rodriguez-López, R. Martínez-Máñez, M. D. Marcos and P. Amorós, Chem. Commun., 2002, 330

11 P1-MS and P1-FS possess a hydrophobic surface with a molar ratio of $\mathrm{CH}_{3} / \mathrm{SiO}_{2}=0.33$ and 0.25 , respectively, and $\mathbf{P 1}$ is incorporated with a molar ratio of pyrylium $/ \mathrm{SiO}_{2}=6.38 \times 10^{-4}$ and $2.28 \times 10^{-4}$ into $\mathbf{P 1}$ MS and P1-FS, respectively. Additional characterization for P1-MS powder X-ray patterns show the presence of a disordered MCM-41 phase indicating that nothing changed after the functionalization process. IR spectra of P1-MS showed silanol bands at $1080 \mathrm{~cm}^{-1}$ and stretching $\mathrm{C}-\mathrm{H}$ vibrations (from $\mathrm{CH}_{3}$ groups) at $2964 \mathrm{~cm}^{-1}$ and bands in the $850-600 \mathrm{~cm}^{-1}$ range due to stretching vibrations of $\mathrm{Si}\left(\mathrm{CH}_{3}\right)_{3}$ moieties. Due to its low relative amount in the final solid, bands of the pyrylium group were not detected. $\mathrm{N}_{2}$ adsorptiondesorption isotherms showed characteristic behaviour for a mesoporous material with a specific surface (BET model) of $753 \mathrm{~m}^{2} \mathrm{~g}^{-1}$, a volume of $0.56 \mathrm{cc} \mathrm{g}^{-1}$ and a pore diameter (BJH method) of $2.51 \mathrm{~nm}$.

12 P1-PVC was prepared by mixing P1 ( $2 \%$ wt.), poly(vinyl chloride) (49\%wt.) and nitrophenyl octyl ether (49\%wt.) in $5 \mathrm{~mL}$ of THF. The solvent was evaporated to give a blue-greenish membrane.

13 In preliminary studies we have observed that the sensory materials P1FS, P1-MS and P1-PVC can in part be regenerated after their use by treatment with an acidic aqueous solution that partially liberates the original blue pyrylium probe. We will address this reactivity in the future, based on the detailed reaction mechanisms of pyryliumpyridinium interconversion [see for instance ref. 5: A. T. Balaban et al. pp. 114-115]. This reversible process points towards the possibility of using the sensory materials for repetitive cycles for qualitative purposes. 